\title{
Understanding Dual Diagnosis: Substance Abuse and Mental Illness
}

\author{
Jackson De Carvalho ${ }^{1 *}$ and Darron Garner ${ }^{2}$ \\ ${ }^{1}$ Associate Professor, Prairie View A \& M University, USA \\ ${ }^{2}$ Department Head for Human Services, Alvin Community College, USA
}

*Corresponding author: Jackson De Carvalho, Associate Professor, Prairie View A \& M University, USA.

Received Date: July 18, 2019

Published Date: August 14, 2019

\begin{abstract}
Persons with co-occurring substance use disorder (SUD) and mental illness are referred to as having a dual diagnosis. Individuals with this condition are often very complex and require challenging treatment procedures. In order to improve recovery outcomes for this population, dual diagnosis treatment must be informed by evidence-based practice and research. In order to improve patient care experiences and outcomes, dual diagnosis care must be informed by relevant evidence. Although National Institute of Health [1], released findings showing there are over 8 million people with mental illness and substance abuse in the United States of America (USA), empirical research and evidence-based practice to inform effective care delivery are lacking. There is even less evidence concerning the correlates of dual diagnosis in in-patient settings; including the relationship between mental health service settings and the initiation and maintenance of substance abuse. The purpose of this study is to explore the correlation between the drug of choice and psychiatric problems while examining the prevalence and social context for substance use among those with psychotic illnesses. The term dual diagnosis describes the co-existence of two disorders and has been used as a label for several medical and psychiatric conditions, e.g., learning disability and mental illness, personality disorder and depressive illness. More recently, it has been commonly used as a label to describe people with mental health problems who also have a substance use disorder.
\end{abstract}

Keywords: Substance abuse; Mental illness; Dual diagnosis; Evidence and research

\section{Introduction}

The use and misuse of alcohol and illicit drugs has increased significantly since the new millennium across many communities, cultures, social class groups, age groups and in different parts of the world [2]. There is a high rate of substance abuse among those with mental illness, and there is a high rate of mental illness among those who abuse substances. Research evidence suggests that some people with mental health problems have participated in this general trend of increased substance use. The phenomena of and addiction are very complex, in part due to the obscurity of drug abuse and the co-occurrence of mental illness. Opiates, stimulants, sedatives, and hallucinogens produce a unique series of pharmacological effects on the brain. Hedden SL [3] identifies this by suggesting that the year of a report of prevalence data of dual diagnosis is highly correlated to the actual prevalence rate reported; the later the report, the higher the prevalence rate reported in the study. Some individuals with mental health problems have then gone on to develop substance use disorders, and this phenomenon has been called dual diagnosis $[1,4]$.
Persons with co-occurring substance use disorder (SUD) and mental illness, are often referred to as having a dual diagnosis [5]. Individuals with this condition are often very complex and require challenging treatment procedures [6]. In order to improve recovery outcomes for this population, dual diagnosis treatment must be informed by evidence-based practice and research [6]. In order to improve patient care experiences and outcomes, dual diagnosis care must be informed by relevant evidence [5]. Although the National Institute of Health [1] released findings showing there are over 8 million people with mental illness and substance abuse in the United States of America (USA), empirical research and evidence-based practice to inform effective care delivery are lacking. There is even less evidence concerning the correlates of dual diagnosis in in-patient settings; including the relationship between mental health service settings and the initiation and maintenance of substance abuse. Critically, there is no single diagnostic criterion for dual diagnosis, as it is not a homogenous syndrome and there is no consistent set of symptoms or illness 
course, but rather there is a wide range of patterns and types of substance use, effects on symptoms and prognosis. The term dual diagnosis describes the co-existence of two disorders and has been used as a label for a number of medical and psychiatric conditions, e.g., learning disability and mental illness, personality disorder and depressive illness. More recently, it has been commonly used as a label to describe people with mental health problems who also have a substance use disorder $[7,8]$.

Clinically, however, it appears that the psychiatric disorder and the specific substance of abuse are not randomly associated; the association between psychiatric problems and substance abuse is not clear. There is some evidence of specific drugs being associated with specific psychological problems, but not everyone has found these associations [9]. The question, therefore, is whether there is an association between the type of mental illness, or the symptoms manifested, and the type of substance abused. In the presence of a comorbid diagnosis (mental illness and substance abuse), the etiology can be complicated to determine. Since there is limited knowledge of the factors surrounding substance abuse, establishing the function of the relationship is important. It is the theoretical assumption of this study that the drug of choice is a function of and correlated with, the primary psychological symptomatology. Although the symptomatology may drive the drug of choice, it is possible, as others have argued [2], that the drug of use/abuse causes the specific psychological symptomatology. The purpose of this study, however, is to explore the correlation between the drug of choice and psychiatric problems while examining the prevalence, social context of, and motivations for substance use among those with psychotic illnesses.

\section{Literature Review}

According to NIDA [2], 25.8 million people (12 years and older) needed treatment for illicit drug or alcohol use problems, while only 4.6 million obtained such specialized treatment. Further, NIDA also reported that as many as 6 in 10 persons with an illicit substance use disorder also had another mental illness. Nevertheless, the Substance Abuse and Mental Health Services Administration [10], has recognized that screening for co-occurring mental health and substance abuse is complicated and probably results in the underidentification of persons with a dual diagnosis. Further, the office of Substance Abuse and Mental Health Services Administration [11], reported that persons who use and abuse drugs are more likely to have mental disorders. The Treatment Episode Data Set Report (TEDS), a division of SAMHSA, reported that among older substance abuse treatment admissions (aged 50 or older) multiple substance abuse has nearly tripled [11-13].

Research studies investigating substance abuse disorders have documented the co-occurrence of psychiatric disorders in clinical samples population-based studies. Among the chronically mentally ill the occurrence of a comorbid substance use is generally high, with estimates ranging from $30 \%$ to $50 \%$ and varying depending on the subgroups being assessed $[3,14]$. In fact, the studies of Greydanus DE, et al. [15], found that empirical epidemiological research, although limited, indicated substantial, variations suggesting that comorbidity, in general, should be addressed universally in the context of local patterns of substance misuse, that consider the influences of social and cultural factors. Co-occurring disorders often vary in severity and impairment of functioning. It is presumed that more than $50 \%$ of all adults with severe mental illness are also affected by substance abuse related to alcohol or other drugs [8]. The Georgia Department of Human Resources found that approximately half of the individuals with severe mental illness are affected by substance abuse, while approximately a third of alcohol abusers and half of all other drug abusers have at least one serious mental illness [11]. A report of National Institute for Health and Care Excellence [16] found that approximately 8\% of persons with either a mood or anxiety disorder abused amphetamines, a slightly higher percentage abused cocaine, while cannabis was abused by more than $32 \%$ of persons diagnosed with a mood or anxiety disorder (mood disorder was slightly higher).

Furthermore, National Institute for Health and Care Excellence [16] estimated that roughly $75 \%$ of offenders in state and local prisons or jails have a comorbid mental health and substance abuse or addiction problem although they did not indicate the percentage of each psychiatric problem and co-occurring disorder. The Epidemiology of Co-Occurring Substance Use and Mental Health Disorders [17], stated that the NSDUH (National Survey on Drug Use and Health) data estimated that approximately 6.6 million people are estimated to have a clinically significant mental disorder with a co-occurring substance use disorder. Most notably, a preliminary finding from this data suggested that the onset of a "diagnosable mental disorder often precedes the onset of a diagnosable substance disorder" [17]. However, one of the difficulties noted with this, and other publications, is that the definition of severe mental disorder is not universally defined. In addition, the NSDUH does not distinguish specific disorder.

According to McClean, et al. [18], prior empirical studies of cooccurring disorders, that address the biopsychosocial dimensions of substance abuse, found limited support for a direct relationship between the psychiatric symptom and drug choice. However, their investigation confirmed the findings of other researchers [18-21] and showed that specific personality characteristics are often linked with specific drug of choice. In other words, a depressive emotion often leads to a higher likelihood of alcohol abuse, and cocaine abuse could be associated with individuals who had difficulty with dysregulation (higher levels of desire for elation and restlessness), while heroin or opiate use are often present in individuals who experience mood or anxiety disorder symptomatology.

\section{The Nosology of Dual Diagnosis}

The literature concerning dual diagnosis has mostly identified and defined the mental health aspect of it as the presence of a functional psychotic illness such as schizophrenia, manicdepressive psychosis, delusional disorder, schizoaffective disorder, depressive illness with psychotic symptoms, but is less specific when identifying the substance use aspect of dual diagnosis. It 
can be argued that this definition is excessively narrow since it excludes those patients with nonpsychotic mental health problems from the potential benefit of any specialist help or evidence-based intervention available to those with substance use disorders and functional psychotic illness $[3,6,18,22]$.

It is possible that the patients' self-identity and primary service engagement are critical factors relating to the limitations associated with the definition of dual diagnosis, as many studies $[19,18]$ identified high levels of psychological and psychiatric morbidity in drug users, and high levels of substance use among general psychiatric patients with personality disorder, depression and anxiety. However, these arguments concerning definition can be contrasted against the relative merits of the label of dual diagnosis since there is a limited clinical or academic consensus about what actually constitutes dual diagnosis, and there is as yet only a limited evidence base concerning which forms of treatment and therapeutic intervention work most effectively for those with this combination of diagnoses $[23,24]$. It is noteworthy that an extensive review of the current literature concerning the dual diagnosis phenomenon has mostly identified and defined the mental health aspect of dual diagnosis as the presence of a functional psychotic illness such as schizophrenia, manic-depressive psychosis, delusional disorder, schizoaffective disorder, depressive illness with psychotic symptoms, but is less specific when identifying the substance use aspect of dual diagnosis.

\section{Substance use disorders}

According to Tulloch, et al. [25], the terminology used to describe substance disorders are based on diagnostic language, while others have a pejorative meaning (Junky, addict, misuser). Within the two psychiatric classification systems, the Diagnostic and Statistical Manual of Mental Disorders, 5th edition (DSM-5) and the International Classification of Diseases, 10th revision, Clinical Modification (ICD-10-CM) include significant groups of diagnoses, which relate to ongoing severe and problematic drug and alcohol use; these are harmful use and the dependence syndrome. The ICD10 describes harmful use as a pattern of psychoactive substance use that is causing damage to the individual's health. The damage may relate to either physical health (as in the case of hepatitis from injection drug use behavior) or mental health (as in depressive disorder secondary to heavy alcohol use), or indeed both. The ICD10 identifies four main criteria for harmful use;

- Clear evidence that the substance consumed was the cause for the physical or psychological harm,

- A clear and identifiable nature of the harm.

- $\quad$ The pattern of use has persisted for at least one month, or has occurred repeatedly within a twelve-month period, and

- $\quad$ The disorder does not meet criteria for any other mental or behavioral disorder related to the same drug in the same time period, except for acute intoxication (National Institute for Health and Care Excellence [26].
Dependence syndrome on the other hand, comprises a cluster of behavioral, cognitive, and physiological phenomena that develop after repeated substance use, and usually include a robust subjective desire to take the substance, struggle in controlling its use despite of the consequences, a higher priority given to substance use, increased resistance and sometimes physical withdrawal states. Presence of increased dependence to a specific psychoactive substance (e.g., diazepam), or for a class of substances (e.g., opiates), or for a wide range of pharmacologically different substances [5].

The ICD-10 [27] identifies six main criteria for dependence, of which three must be present for a period of at least one month:

- A robust subjective desire or compulsion to take the substance,

- Impaired capacity to control substance taking behavior in terms of its onset, termination, or levels of use, as demonstrated by the substance being used for an extended period of time and in large amount,

- A significant withdrawal when reducing or stopping the use of substance of choice, followed by continuous substance in order to relief or avoid withdrawal symptoms,

- Clear tolerance evidence to the effects of the substance being used

- A preoccupation with substance use,

- $\quad$ Consistent use of substance of choice with total disregards of harmful consequences of such use of which the individual is subjectively aware.

\section{Socio-Demographic and Clinical Characteristics of Patients with Dual Diagnosis}

Substance use problems and disorders are reported more commonly in male patients [13]. In fact, Lechliter, [4] reported male patients with psychotic illness in a South London study were more likely to have a substance misuse problem than their female counterparts, reflecting gender differences in the reported rates of substance misuse in the general population. However, the underrepresentation of women in substance misuse treatment services is well documented $[28,29]$ and it may be that lower reported rates of substance misuse in women reflect women's reluctance to disclose substance misuse, or seek help from treatment services because of more significant stigma, and/or fear that any children may be removed, thereby creating artificially low prevalence and incidence of dual diagnosis in women. Patients with a dual diagnosis tend to have an earlier onset of psychotic illness than patients who do not use substances [21].

Degenhardt, et al. [30] reported that the premorbid characteristics of patients with dual diagnosis are often different from those with psychosis alone. Childhood behavioral problems have been found to have been more common among psychotic patients who did not misuse drugs. Possibly related to these findings 
are reports of better premorbid personality, social adjustment, and interpersonal relationships in people with schizophrenia and substance misuse than those with schizophrenia only [31]. A plausible explanation is that those pre schizophrenic individuals who have demonstrated abnormal childhood behaviors require little additional stress to develop a frank psychotic illness in adult life; whereas those with little or no abnormal childhood functioning are likely to need more in the way of risk factors including drug and alcohol misuse before they become psychotic [5]. Additionally, those schizophrenic patients who did experience childhood functioning problems are less likely to have acquired the social skills necessary first to be exposed to the substance misuse culture and then to negotiate the drug market [30].

\section{Drug of Choice and Comorbidity}

There is a gap in the existing body of research focused on the association of specific substances of abuse and their relationships to mental disorders. It is important to explore such correlations and to ascertain if there is a relationship between the substance of abuse and specific type of psychiatric disorder in order to gain a better understanding of the comorbidity. With this understanding, of which this is the first step, better treatments can be designed for the patients and, depending on the extended results, and perhaps something can be done about the frequencies of specific substances of abuse among the mentally ill or perhaps the frequencies of mental illnesses among those abusing substances.

\section{Alcohol}

The research literature abounds with studies exploring the relationship between depression and alcohol use [7]. Although correlation does not presume causation [32] found a "high functional relationship between alcohol dependence and depression" in a clinical sample of alcohol-dependent adults [32]. In a study of Alcoholics Anonymous (AA) [33] found that higher AA attendance was associated with a greater reduction in symptoms of depression [33]. The authors also said that this relationship between alcohol consumption and depressive symptoms supported the role of Alcoholics Anonymous in remittance of depression symptoms, as well as lowering the frequency and intensity of alcohol consumption, although it did not predict additional improvements in depression [33].

Alcohol abuse has been linked to social anxiety disorder (SAD), which is also known as social phobia. SAD is characterized by a significant amount of fear of being judged in social performance situations, which often cause stress and dysfunction. Comorbid substance use disorder (alcohol) and anxiety disorders, particularly generalized anxiety disorder, has a lifetime prevalence rate ranging from 2-4 \% in Western societies [31,34]. One study [35], found that the onset of a generalized anxiety disorder predates an alcohol use disorder by more than a decade; its comorbidity with alcohol is $67 \%$. Alegria et al [31]. report a worse outcome for this comorbidity. Their findings "suggest a stronger predisposition for psychopathology among individuals with GAD-SUD (generalized anxiety disorder, substance use disorder, i.e., alcohol) that is further exacerbated by the use of substances." [31]. A generalized anxiety disorder, among individuals with alcohol use disorder, dramatically increases the risk of a recurrence of the anxiety disorder. It also decreases the likelihood of recovery from an anxiety disorder. The symptoms present in this comorbidity are more severe and prolonged than when found in isolation: greater hospitalizations, greater severity of psychiatric illness, higher consumption rates of alcohol, and worse social adjustment $[31,35]$.

The presence of a comorbid social anxiety disorder and alcohol use disorder, according to [36], varies from 15 to $20 \%$ depending on the symptom presentation and treatment received. Alcohol use is reported as an elixir to stress reduction of social fears, a negative reinforcer, eliminating or reducing stress. Alcohol consumption in this population can also vary depending on the stressors present and the intermittent ability of the individual to determine or seek social situations that do not present stressors that activate a social anxiety disorder. Just as a biopsychosocial model of addiction disorders support a multidimensional view of substance abuse, understanding alcohol's role in mood disorders, versus anxiety disorders, needs to be illuminated [3]. It is noteworthy that in the relevant research study literature, alcohol use disorder (AUD) is present as a covariant with anxiety disorders, most notably generalized anxiety disorder (GAD). According to [37], "in general, the effects of alcohol intoxication follows a biphasic time course as the initial feelings of relaxation and exuberance give way to ... depression". Therefore, considering AUD within the realm of mood rather than anxiety disorders necessitates an examination of the multiple effects and interaction of ethanol, the significance of comorbid factors, with a neurobehavioral effect, ultimately resulting in an increased depressive affect [37]. In other words, the anxiety-prone individual will abuse alcohol to meet a specific need (stress or tension reduction), whereas the mood-disordered individual will depend on alcohol to achieve the feeling of normalcy.

The research study of [38] posited a tension reduction theory to explain the comorbidity between social anxiety disorders and AUD. They assumed that people with social anxiety use alcohol to alleviate their fears. Their findings that individuals with clinically diagnosed social anxiety disorder had a higher incidence of alcoholrelated difficulties than that of the general population presumes that persons with social anxiety use alcohol to alleviate their symptoms of anxiety and alleviate their fears. The self-medicating hypothesis [39,19], according to [33], found that the relationship between alcohol consumption and depressive symptoms supported the role of Alcoholics Anonymous in reduction, but not remission of depression symptoms. In addition, [31] found that individuals with substance use disorder showed a higher prevalence of lifetime psychiatric comorbidity than those without a substance use disorder.

\section{Cannabis/Hallucinogens}

Cannabis is among the most commonly used controlled substances worldwide [25, 40-42]. It is also rapidly becoming the primary source of drug problems for all new treatment seekers, 
including treatment for individuals with comorbid disorders [10]. The studies of $[43,44]$ found that long-term cannabis use to be associated with deficits in attention in chronic and mature users as well as in short-term and working memory. Additionally, [45] found that acute intoxication with cannabis resulted in marked changes in neuropsychological performance, including subjective mental status and brain functioning. More recent studies of longterm cannabis users suggest that neuropsychological impairments such as memory and attention may be due to brain alterations from cannabis abuse. Thus, determining the effects of cannabis use on brain functioning and the resulting neurobehavioral sequelae of a cannabis-use disorder may be predicated upon innate cognitive abilities [41]. Cannabis is the most widely used mind-altering and controlled substances worldwide. A more recent concern for prolonged cannabis abuse is the physiological and neurobiological functions observed [45]. More than any other substance of abuse, cannabis is associated with the development of psychosis; however, the temporal relationship is still to be determined $[40,46]$. Longterm cannabis use is generally considered a precipitant and causal factor for a minority of individuals, although dose dependency, early onset of use, and other confounds [47] may assume that cannabis use increases risk of a psychiatric disorder, primarily among vulnerable individuals so predisposed. In fact, [48] found that for diagnosed schizophrenic patients, symptom severity was only marginally increased with continued substance abuse.

According to [48], cannabis dependence was more highly associated with psychiatric treatment than comorbid use of any other substance. Additionally, a new treatment for dependence on cannabis was more highly associated with previous treatment for a psychiatric disorder [46] Cognitive impairment found in chronic cannabis use may also limit its effectiveness in treatment outcomes. [42] reported that "impaired cognitive functioning predicts poor treatment response in marijuana users" suggesting that negative psychological and functional symptoms may be precursors which may, in fact, be the reason for the abuse as well as the high incidence of relapse after treatment. [44] suggests that individuals use cannabis (and other drugs) to regulate their affective state. In other words, a person with a thought disorder could be attracted to cannabis because of its self-medicating properties, and, therefore, could a self-medication hypothesis assume that the psychopharmacological choice of a thought disorder is necessarily cannabis? Proponents of such a causal chain presume psychosis to be a risk factor although the temporal relationship is as yet undetermined. [19,46] found that a history of psychiatric treatment was associated with an increased rate of subsequent treatment for cannabis abuse. In diagnosed schizophrenic patients, symptom severity was, however, only marginally increased with continued substance abuse [48]. Other studies [41] have reported that cannabis abuse was the most significant predictor of nonadherence in patients with first-episode psychosis. [47] found negative psychological symptoms linked to increased relapse use among current marijuana users; however, the frequency of use also correlated with relapse. This correlation was not found with cocaine, suggesting specificity of substance use and psychological disorder. One of the three plausible hypotheses for the association of cannabis and a possible association with a schizophrenia spectrum disorder, that patients with a thought disorder are attracted to cannabis for its self-medicating properties, is not conclusive, even though cannabis was the most frequently used drug $[41,46]$. In fact [44] concluded that a causal relationship could be established, although common vulnerabilities (another hypothesis) and difficulties establishing a temporal relationship could provide findings that support a bi-directional causal relationship.

\section{Opioids/heroin}

The causal connection and temporal ordering of psychiatric disorders and substance abuse have important implications for addiction research in general. For opioid abuse and mental disorders, this causal pathway, although yet undetermined [49], is receiving increased attention. According to [34], the most commonly prescribed medication of any category in the United States is an opiate derivate (hydrocodone), its non-medical use a growing problem. Opiate is a term reserved for the chemical compound found naturally in nature, one that is extricated from the opium poppy, Papaver somniferum $[43,50]$. Opioid(s) generally refers to the exogenous drugs - natural or synthetic - that are modified from a natural form or synthetically derived. Endogenous opioids, such as the endorphins, exist naturally in the body. Opiate receptors are found in many regions of the brain and spinal cord; the highest concentration is in the limbic system and other areas that modulate emotional behavior, pain perception, and reward saliency. Injecting, sniffing, or smoking opiates activates opiates receptors resulting in changes in affect state, including euphoria, resulting in "a relaxed, contented, state" [50]. Thus, it would appear that opiate addiction would also be related to a need to reduce anxiety, feelings of tension, unreasonable worries, and the inability to feel relaxed.

Although the research examining anxiety among opioid users (heroin in particular) is limited [51], there is a high rate of comorbidity. In a self-medicating model [19,39] this causal pathway is presumed to initiate with psychiatric distress. Studies of anxiety and General Anxiety Disorder and social anxiety [51] found comorbid opiate abuse to be associated with reported symptoms of anxiety. [43] reported that anxiety disorders commonly cooccur with opiate dependence, but the lack of evidence to support this association is due to the paucity in prospective studies that adequately examine this association. The [27] found that the presence of a mental disorder remained a significant predictor of opioid use. However, this six-fold increase in prescription use could also be attributed to high levels of pain, self-reported pain that is commonly associated with psychological distress, possibly presented as anxiety - the authors propose that opioids are being used to treat this undifferentiated state of mental and physical pain. It is generally considered that exogenous opioids modulate and elevate effect. Martins et al. (2009) [49] found that psychiatric disorders, including anxiety, were three times as likely to precede 
nonmedical opioid use. Their findings stated support for a general vulnerability model as well as evidence for a self-medication model of dependence for General Anxiety Disorder in particular.

[51] reported that previous studies found social anxiety common among opioid users. The authors found that social anxiety is an "under-acknowledged" phenomenon within opioiddependent populations [10,51]. Their research found childhood adversity, specifically parental and peer rejections, to be risk factors for both opioid dependency and social anxiety. Thus, according to [51], an underlying general vulnerability, as well as a need for self-medication could be attributed to this comorbid disorder. The potential for abuse among opioid prescription users is much higher when there is a comorbid mental health diagnosis such as anxiety, particularly when the mental health disorder precedes the substance abuse [52]. [52] cautioned against promoting abuse iatrogenically, reporting that their epidemiological data suggest a temporal order where a mental health disorder precedes substance abuse.

\section{Stimulant, methamphetamine}

The etiology of cognitive impairment in stimulant abuse, in particular, methamphetamine, is recorded in the relevant literature; some studies report that "the rates of impairment correlate with frequency or amount of use observed in several psychiatric symptomatology" [53]. Methamphetamine is a "potent, addictive psychostimulant that has dramatic effects on the central nervous system" [53]. Its desired effect for the addict is, among others, enhanced energy, alertness, increased physical and mental effort, as well as a surge in productivity. At lower doses, these effects apparently can occur. [59] found dysregulation of the orbitofrontal cortex in methamphetamine abusers that has previously been noted in cocaine abusers. Disruption of functioning in this area is related to dysregulation of behavior, including obsessive and compulsive behavior. For the individual with dysregulation, the initial psychostimulant behavioral effect would appear to enhance functioning related to an attentional disorder. In addition, the desired cognitive and emotional effects of methamphetamine include increased selfesteem and an increase in libido [53]. Related to this, [54,55] stated that there appear to be no studies examining the link between neurocognition and functional outcomes in methamphetamine addiction. However, they do add that the neurocognitive impairments appear to be irreversible.

Long-term methamphetamine abuse or dependence presents physical and mental health consequences, including the wellreported "meth-mouth" (i.e., rapid tooth and gum decay) so often seen in chronic users. Presently, although it is available by prescription for narcolepsy, obesity, and treatment of attention deficit disorder, methamphetamine is frequently obtained by the recreational user, which may attest to its role in managing difficulties related to executive functioning. Chronic users also have high rates of psychiatric symptoms and affective distress known to be especially acute during withdrawal. Craving as a construct is still a matter of debate within clinical research. [56,57] examined the role of metacognition in suppressing cravings. They found that the internal strategies and cognitive processes that control cravings had a great deal to do with relapse prevention, which may also address the role amphetamines play in assisting executive functioning. The presence of a psychotic disorder that is chemically induced, often seen in the recreational user, is usually transient, its presence determined by substance use and or abuse. However, the differential diagnosis must carefully consider the possibility of an exogenous drug induced diagnosis versus the presence of psychopathy, particularly difficulties that relate to dysregulation of function found in ADHD, among others. In addition, [58] found methamphetamine users to demonstrate aggressive behaviors and increased impulsivity, behavioral traits that could be presumed to be part of a "broader personality dimension" [58] not merely attributed to the substance of abuse. [58] found both positive and negative symptom psychosis in methamphetamine-abusing individuals in far more significant proportion than found in the general population.

\section{Stimulant, cocaine}

The possible irreversibility in the structural alterations in the cerebral morphology of cocaine users/ abusers has been well documented in the current literature. Remission of brain changes (neuronal) with prolonged abstinence as has been extensively reviewed in alcohol studies, have been completed for cocaines, and it is known that "brain changes observed will remit with prolonged abstinence" [53]. According to [59], among chronic cocaine abusers, deficits have been found in areas related to executive functioning (ability to attend, organize, and integrate information); found that those with cocaine addiction have lower frontal lobe activation. Their research into brain activation associated with drug craving found that those with cocaine addiction have less activation when compared to healthy subjects in multiple areas within the frontal lobes. [60] found that long-term cocaine use, from a psychiatric perspective, has aspects of both impulse control and a compulsive disorder characterized by a dysregulation of frontal brain reward pathways. Research on executive functioning impairment and addictive behavior [61] found impairment in decision-making processes. Whether this drives the addiction or is a result thereof is yet to be determined. According to [2], methamphetamine abuse and addiction lead to significant brain changes, which often occurs following chronic abuse, in particular areas involved with the dopamine system (e.g., motor speed, verbal learning). [59] found functional impairments most at risk related to memory and attention for chronic users of cocaine. However, functional impairment can also be affected by previous psychiatric problems. [62] found that even with significant abstinence, amphetamine-dependent users exhibit impairments in verbal memory that appear related to poor organizational strategies. [59] found abstinence in cocaine-abusing methadone patients to be more successful in those who responded early in treatment, perhaps indicative of psychopathy related to dysexecutive functioning.

Some studies postulate that it is the impairment that drives the addiction -- the failure of a supervisory control mechanism affects 
the nature and quality of decision making -- while others assume that the addiction results in impairment. However, determining the limitations found does not preclude their presence before addiction. In fact, difficulties with attention are one of the hallmarks for drug administration for an attention deficit disorder. The comorbidity of stimulant abuse and dysregulation of executive functioning is a complex phenomenon related to the neuropsychological and neurobiological brain/behavior phenomenon. The etiology of this cognitive impairment and stimulant abuse has yet to be determined. It has been postulated that the desired effect of increased energy, alertness, and mental/physical effort increases the need for increased use leading to substance abuse [53]. Others posit that chronic and long-term prescribed use leads to difficulties already present in neurocognitive functioning. The presence of a psychotic disorder that is chemically induced, at present, is assumed to be transient and reversible. However, the behavioral characteristics of neurocognitive impairments and dysregulation of executive functioning [59] impair decision making processes, which may drive the addictive process.

\section{Heroin}

On the other hand, Heroin use is on the increase; its highest rate of usage purported among individuals who are just entering adulthood [50]. In the prison population, heroin abuse and dependence are much higher than in the general population [61]. While incarceration appears to place an individual in a high-risk environment for both initiating and maintaining substance abuse, more than a third of convicts with a lifetime heroin use reported continued use while incarcerated [27]. This is in contrast to either lifetime cocaine or amphetamine users, of which only $11 \%$ of cocaine and $5 \%$ of amphetamine users continued their use while in prison. Additionally, most of the individuals who were prescribed methadone continued to use heroin while incarcerated [34]. Mental health problems are also reported to occur with higher frequency within the prison population than the general population. Rettner reported that individuals with a psychiatric diagnosis were also more likely to have initiated drug use, heroin in particular, while incarcerated. This increased heroin use might be attributed to its availability. It could also be presumed that given the illegal nature of drug use, the crime related to its distribution, and the environment in which it is consumed, this preponderance of substance abuse within the prison population could be expected. Heroin abuse remains a significant concern; even prior prison experience increases the odds of heroin use [29,63].

However, the preponderance of heroin use may be determined by its effects on the user, for as with the reports from Vietnam $[29,63]$, heroin has been considered as a deterrent against boredom or an effective agent in allaying stress. What has yet to be determined is who is most at risk for its continued use beyond the confines of a given environment or situation. Continued use would appear to relate to mental health factors of which stress reduction and anxiety appear integral. The relationship of heroin abuse and incarceration appears to relate to the properties of the drug; however, its availability also speaks to the preponderance of use/abuse (in contrast to stimulants) within the prison population. Gender differences were also found in the abuse of prescription opioids, in particular, as related to anxiety symptomatology and a history of previous abuse [64]. However, early physical and sexual abuse resulting in comorbid anxiety disorders and opiate abuse were not found to be gender specific [65]. Resilience and risk factors also affected comorbidity.

\section{Implications for Practice}

Wellness can be considered from the perspective of how individuals perceive, among others, their own choice of health and self-care [1]. Providing appropriate intervention and successful treatment for those who have mental illness and a co-occurring substance disorder necessitates focusing on "treating those vulnerabilities that are driving the individual's choice of abuse" [66], namely their specific mental disorder. Until recently, much of intervention has focused on the treatment of either a substance or a mental disorder with success determined by changes within the treatment of a unitary, diagnosis specific treatment plan [67]. It is noteworthy [21] found coping strategies to affect abstinence and relapse of substance abuse (heroin). Nonetheless, what this and many other studies have not considered are the confounding variables, specifically the presence of a mental disorder. In fact, "Berkson's bias" suggests that addicts in treatment may be the least likely to stop using drugs" [68], which the author attributes to the co-occurrence of additional disorder(s). According to [68], "the most important comparison of successful treatment outcome is that addicts in recovery are more likely to suffer from additional psychiatric disorders than those not in treatment". Subsequently, "addiction is actually a self-medication process". Additionally, "addicts use drugs to solve their pre-drug psychological problems" [68], which is also supported by Di Lorenzo (2014) [5,69]. However, [68] asserts that viable alternatives to drug use - the addict's ability to "take advantage of nondrug competing activities" is what determines successful treatment outcomes. Mate [69], instead, assumed that the reasons for the addiction are "rooted in the neurobiology and psychology of emotions".

Specifically, he feels emotional isolation, powerlessness, and stress are critical. For, according to [69], "drugs, in short, do not make anyone into an addict, any more than food makes a person into a compulsive eater. There has to be a preexisting vulnerability" [69]. Further, Mate felt that three things were needed for addiction: a susceptive organism, a drug with addictive potential, and stress. It is the possibility that the drug addiction is a "chronic brain condition" [69], an altered and dysfunctional brain that utilizes an exogenous chemical in an attempt to overcome its own dysfunction and to assume an appearance of normalcy. Therefore, "addiction may not be a natural state, but the brain regions it subverts are part of our central machinery of survival" [69-72]. For a minority of individuals who are not able to overcome drug dependence and for those diagnosed with a co-occurring mental illness, the continued use of an addictive substance may serve as an adaptive, albeit misguided, attempt at normalcy. 


\section{Conclusion}

Substance misuse is a common problem among users of mental health services, which intimates creative and practical solutions to address these problems. Although it was uncommon for individuals to medicate the symptoms of their illness with substances directly, their motivations for use reflected a range of social difficulties, isolation, and other active problems. Further investigation of demographic variables and substance use motivations in a larger sample may be an effective way of delineating subgroups with distinct motivations and of developing treatment strategies which take these motivations into account. Furthermore, it could be argued that the philosophical differences between substance abuse treatments and mental health therapies negatively influence the course of an individual's substance use disorder. Such differences often result in barriers to obtaining treatment and contribute to poor treatment engagement, high dropout rates, and relapse. Since many programs fail to integrate treatment approaches, the client is entirely responsible for the integration. The client often fails in this situation and is considered difficult or labeled as treatment resistant. Clearly, a need for mandatory substance abuse training among practitioners exists, to bridge the disconnect between what science has revealed about substance abuse and what practitioners perceive to be true about it.

\section{Acknowledgements}

None.

\section{Conflict of Interest}

No conflicts of interest.

\section{References}

1. National Institute of Health (2015) Dual diagnosis. National Library of Medicine United, US

2. National Institute on Drug Abuse (NIDA) (2018) Comorbidity: Addiction and other mental illnesses. NIDA Research Report Series, US.

3. Hedden SL (2015) Behavioral health trends in the United States: Results from the 2014 national survey on drug use and health.

4. Lechliter L (2009) Insecure attachment styles, Cluster B Personality Disorders, and gender as risk for substance abuse diagnosis. Fielding Graduate University, US.

5. Di Lorenzo R, Galliani A, Guicciardi A, Landi G, Ferri P (2014) A retrospective analysis focusing on a group of patients with dual diagnosis treated by both mental health and substance use services. Neuropsychiatr Dis Treat 10: 1479-1488.

6. Hunt GE, Siegfried N, Morley K, Sitharthan T, Cleary M (2013) Psychosocial interventions for people with both severe mental illness and substance misuse. Cochrane Database Syst Rev 10

7. Johnson M (2012) Bibliotherapy and journaling as a recovery tool with African Americans with substance use disorders. Alcoholism Treatment Quarterly 30: 367-370.

8. Lickerman A (2010) Co-occurring disorders. Psychology Today.

9. Ponizovsky AM, Rosca P, Haklai Z, Goldberger N (2015) Trends in dual diagnosis of severe mental illness and substance use disorders, 19962010, Israel. Drug Alcohol Depend 148: 203-208.

10. Substance Abuse and Mental Health Services Administration (SAMHSA) (2018) Recovery and recovery support.

11. Substance Abuse and Mental Health Services Administration (SAMHSA)
(2017) Recovery and recovery support

12. American Psychiatric Association (APA) (2019) Diagnostic and statistical manual of mental disorders: DSM-5. In: Arlington VA.

13. Lambert B, Scheiner M, Campbell D (2010) Ethical issues and addiction. J Addict Dis 29(2): 164-74.

14. Gil Rivas V, Prause J, Grella C (2009) Substance use after residential treatment among individuals with co-occurring disorders: The role of anxiety/depressive symptoms and trauma exposure. Psychol Addict Behav 23(2): 303-14

15. Greydanus DE, Kaplan G, Patel DR, Merrick J (2014) Substance abuse. International Public Health Journal 6: 215.

16. National Institute for Health and Care Excellence (2016) Coexisting severe mental illness and substance misuse: community health and social care services. England.

17. Center for Substance Abuse Treatment (2007) The epidemiology of cooccurring substance use and mental disorders. COCE, Overview Paper 8.

18. Mc Clean JM, Anspikian A, Winters BN, Tsuang JW (2014) Factors that affect treatment initiation among individuals with serious mental illness and substance abuse disorder. Addictive Disorders and their Treatment 13: $16-24$.

19. Khantzian E (2005) New windows on understanding addictive vulnerability. Psychoanalytic Dialogues 15: 613-619.

20. Merlo LJ, Trejo-Lopez J, Conwell T, Rivenbark J (2013) Patterns of substance use initiation among healthcare professionals in recovery. Am J Addict 22(6): 605-612.

21. Rani S, Byrne H (2014) 'Telling their stories' on a dual diagnosis training course: Forensic mental health service users' perspective on their challenges, benefits and future strategies. Nurse Educ Pract 14 (2): 200207.

22. Mc Govern MP, Lambert Harris C, Gotham HJ, Claus RE, Xie H (2014) Dual diagnosis capability in mental health and addiction treatment services: An assessment of programs across multiple state systems. Adm Policy Ment Health 41(2): 205-214.

23. Woodhouse R, Neilson M, Martyn St James M, Glanville J, Hewitt C (2014) Interventions for drug-using offenders with co-occurring mental illness. The Cochrane Database of Systematic Reviews 1.

24. Rani S, Byrne H (2012) A multi-method evaluation of a training course on dual diagnosis evaluation of dual diagnosis training course. J Psychiatr Ment Health Nurs 19(6): 509-520.

25. Tulloch AD, David AS, Thornicroft G (2015) Exploring the predictors of early readmission to psychiatric hospital. Epidemiol Psychiatr Sci 25(2): 181-93.

26. National Institute for Health and Care Excellence (2011) Psychosis with coexisting substance misuse: Assessment and management in adults and young people. UK.

27. Centers for Disease Control and Prevention (CDC) (2011) Vital signs: Overdoses of prescription opioid pain relievers-United States 19992008. MMWR, 60: 1487-1492.

28. Back SE, Payne RL, Simpson AN, Brady KT (2010) Gender and prescription opioids: Findings from the National Survey on Drug Use and Health. Addict Behav 35(11): 1001-1007.

29. Maremmani I, Stefania C, Pacini M, Maremmani AG, Carlini M, et al. (2010) Differential substance abuse patterns distribute according to gender in heroin addicts. J Psychoactive Drugs 42(1): 89-95.

30. Degenhardt L, Chiu WT, Conway K, Dierker L, Glantz M, et al. (2008) Does the "gateway" matter? Associations between the order of drug use initiation and the development of drug dependence in the National Comorbodity Study Replication. Psychol Med 39(1): 157-167.

31. Alegria AA, Hasin DS, Nunes EV, Liu SM, Davies C, et al. (2010) Comorbidity of Generalized Anxiety Disorder and Substance Use Disorder: Results from the National Epidemiological Survey on Alcohol and Related Conditions. J Clin Psychiatry 71(9): 1187-1195. 
32. Manwell LB, Czabala JC, Ignaczak M, Mundt MP (2004) Correlates of depression among heavy drinkers in Polish primary care clinics. Int J Psychiatry Med 34(2): 165-178.

33. Kelly JF, Stout RL, Magill M, Tonigan JS, Pagano ME (2010) Mechanisms of behavior change in Alcoholics Anonymous: Does Alcoholics Anonymous lead to better alcohol use outcomes by reducing depression symptoms? Addiction 105(4): 626-636.

34. Addiction Center (2017) College Drug Abuse. Addiction Center.

35. Edelman EJ, Fiellin DA (2016) Alcohol use. Ann Intern Med 164(1).

36. Mark Jayne, Valentine G (2016) Alcohol-related violence and disorder. Progress in Human Geography 40(1): 67-87.

37. Oscar-Berman M, Marinković K (2007) Alcohol: Effects on neurobehavioral functions and the brain. Neuropsychol Rev 17(3): 23957.

38. Book SW, Randall CL (2002) Social Anxiety Disorder and alcohol use. Alcohol Research and Health, 26, 130.

39. Khantzian E (1995) The 1994 distinguished lecturer in substance abuse. Journal of Substance Abuse Treatment 12(3):

157-65.

40. Ben Amar M, Pharm B, Potvin S (2007) Cannabis and psychosis: What is the link? Journal of Psychoactive Drugs, 39, 131-141.

41. Rodrigo C, Rajapakse S (2009) Cannabis and schizophrenia spectrum disorders: A review of clinical studies. Indian J Psychol Med 31(2): 6270 .

42. Sofuoglu M, Sugarman DE, Carroll KM (2010) Cognitive functioning as an emerging treatment target for marijuana addiction. Exp Clin Psychopharmacol 18(2): 109-119.

43. Fatseas M, Denis C, Lavie E, Auriacombe M (2010) Relationship between anxiety disorders and opiate dependence - A systematic review of the literature: Implications for diagnosis and treatment. J Subst Abuse Treat 38(3): 220-230.

44. Flores P (2004) Addiction as an attachment disorder. Int J Group Psychother 51(1): 63-81.

45. Gonzalez R (2007) Acute and non-acute effects of cannabis on brain functioning and neuropsychological performance. Neuropsychol Rev 17(3): 347-61.

46. Saddichha S, Sur S, Sinha BN, Khess CR (2010) How is substance use linked to psychosis? A study of the course and patterns of substance dependence in psychosis. Subst Abus 31(1): 58-67.

47. Bonn Miller MO, Moos RH (2009) Marijuana discontinuation, anxiety symptoms, and relapse to marijuana. Addict Behav 34(9): 782-785.

48. Degenhardt L, Tennant C, Gilmour S, Schofield D, Nash L, et al. (2007) The temporal dynamics of relationships between cannabis, psychosis and depression among young adults with psychotic disorders: Findings from a 10-month prospective study. Psychol Med 37(7): 927-934.

49. Martins SS, Keyes KM, Storr CL, Zhu H, Chilcoat HD (2009) Pathways between nonmedical opioid use/dependence and psychiatric disorders: Results for the National Epidemiologic Survey on Alcohol and Related Conditions. Drug and Alcohol Dependence 103(1-2): 16-24.

50. Gruber SA, Silveri MM, Yurgelun Todd DA (2007) Neuropsychological consequences of opiate use. Neuropsychol Rev 17(3): 299-315.

51. Shand FL, Degenhardt L, Nelson EC, Mattick RP (2010) Predictors of social anxiety in an opioid dependent sample and a control sample. J Anxiety Disord 24(1): 49-54.

52. Edlund M, Sullivan M, Steffick D, Harris K, Wells KB (2007) Do users of regularly prescribed opioids have higher rates of substance use problems than nonusers. Pain Med 8(8): 647-656.
53. Scott JC, Woods SP, Matt GE, Meyer RA, Heaton RK, et al. (2007) Neurocognitive effects of methamphetamine: A critical review and meta-analysis. Neuropsychol Rev 17(3): 275-297.

54. National Institute on Drug Abuse (NIDA) (2016) Drug abuse and addiction: The basics.

55. Kalechstein AD, Jentsch JD, Kantak KM (2008) Stimulant-associated cognitive abnormalities: Mechanisms and impact on reward-related behavior and addiction. Drug Alcohol Depend 97(3): 276-289.

56. De Matteo D, Filone S, Davis J (2015) Substance use and crime. APA Handbook of Forensic Psychology 1: 325-349.

57. Lee NK, Pohlman S, Baker A, Ferris J, Kay Lambkin F (2010) It's the thought that counts: Craving metacognitions and their role in abstinence from methamphetamine use. J Subst Abuse Treat 38(3): 245-250.

58. Lapworth K, Dawe S, Davis P, Kavanagh D, Young R, et al. (2009) Impulsivity and positive psychotic symptoms influence hostility in methamphetamine users. Addictive Behaviors 34: 380-385.

59. Weinstock J, Rash CJ, Petry NM (2010) Contingency management for cocaine use in methadone maintenance patients: When does abstinence happen? Psychol Addict Behav 24(2): 282-291.

60. Koob G, Kreek MJ (2007) Stress, dysregulation of drug reward pathways, and the transition to drug dependence. Am J Psychiatry 164: 1149-1158.

61. Bowler JL, Bowler MC, James LR (2011) The cognitive underpinnings of addiction. Subst Use Misuse 46(8): 1060-1071.

62. Allen DN, Strauss G, Leany BD, Donohue B (2008) Neuropsychological assessment of individuals with substance use disorders. In: MacNeill Horton A, Wedding D (eds), The neuropsychology handbook $3^{\text {rd }}$ (edn), NY: Springer, New York.

63. Robins LN, Helzer JE, Hesselbrock M, Wish E (2010) Vietnam veterans three years after Vietnam: How our study changed our view of heroin. Am J Addict 19(3): 203-211.

64. Green TC, Grimes Serrano JM, Licari A, Budman SH, Butler SF (2009) Women who abuse prescription opioids: Findings from the Addiction Severity Index-Multimedia Version Connect prescription opioid database. Drug Alcohol Depend 103(1-2): 65-73.

65. Kuhn C, Johnson M, Thomae A, Luo B, Simon SA, et al. (2010) The emergence of gonadal hormone influence on dopaminergic function during puberty. Horm Behav 58(1): 122-137.

66. Redish AD, Jensen S, Johnson A (2008) A unified framework for addiction: Vulnerabilities in the decision process. Behav Brain Sci 31(4): 415-487.

67. Bahrke MS (2012) Performance-enhancing substance misuse in sport: Risk factors and considerations for success and failure in intervention programs. Subst Use Misuse 47(1314): 1505-1516.

68. Heyman GM (2009) Addiction: A disorder of choice. Harvard University Press.

69. Mate G (2010) In the realm of hungry ghosts. North Atlantic Books.

70. Merlo LJ, Trejo-Lopez J, Conwell T, Rivenbark J (2013) Patterns of substance use initiation among healthcare professionals in recovery. Am J Addict 22(6): 605-612.

71. Cooper L (2012) Combined motivational interviewing and cognitivebehavioral therapy with older adult drug and alcohol abusers. Health Soc Work 37(3): 173-179.

72. Stamm ME, Frick WC, Mackey HJ (2016) An analysis of U.S. student drug and alcohol policies through the lens of a professional ethic for school leadership. International Journal of Education Policy \& Leadership 11(1): 1-22. 